\title{
EFICIENCIA SOCIAL Y EDUCACIÓN AFECTIVO-SEXUAL
}

\author{
CARMEN GONZÁLEZ MARSAL*
}

Fecha de recepción: 15 de marzo de 2013.

Fecha de aceptación: 11 de febrero de 2014.

I

EFICIENCIA DINÁMICA Y MORAL SEXUAL

\section{Sociedad e instituciones como órdenes espontáneos}

El estudio de la moral está íntimamente relacionado con el concepto de sociedad y según cómo se entienda esta última, se comprenderá también la moral y las demás instituciones sociales. Reconocer que la sociedad y sus instituciones son órdenes espontáneos, es decir, un conjunto de regularidades en las relaciones interpersonales surgido de la adaptación consuetudinaria y evolutiva de los sujetos que actúan a las circunstancias de cada tiempo y lugar, abre la puerta a una perspectiva completamente diferente de la más extendida que las considera organizaciones, esto es, órdenes creados deliberadamente por una persona o un grupo más o menos numeroso de personas. ${ }^{1}$

\footnotetext{
* Máster de Economía de la Escuela Austriaca por la Universidad Rey Juan Carlos. DEA en Filosofía del Derecho, Especialista en Derechos Humanos y Licenciada en Derecho por la Universidad Complutense.

1 Ya Molinari (2006: 219-220 y 229-232) en 1849 advirtió las diferencias entre dos conceptos de sociedad, a saber, el de organización artificial planificada desde arriba y el de asociación natural surgida espontáneamente. Poniendo en práctica el primero, los científicos o dirigentes se dedican a diseñar la sociedad y crear sus normas, dañando múltiples intereses, mientras que de acuerdo con la segunda forma de entender la sociedad, los científicos se limitan a estudiar la realidad social y tratan de descubrir sus leyes, respetando en todo momento la libertad personal de los miembros de la sociedad.

En la misma línea, Mises (2011: 234-238) diferencia la sociedad basada en vínculos contractuales de la de vínculos hegemónicos. Hayek (2006 y 2011) es quien diferencia
} 
El orden espontáneo, sobre la base de unas normas generales abstractas surgidas a lo largo de dilatados periodos de tiempo sin haber sido directamente buscadas, facilita el libre ejercicio de la acción humana — que cada uno persiga sus propios fines-y así permite el mejor aprovechamiento posible del conocimiento disperso en la sociedad. La organización, por el contrario, tiene unos objetivos concretos establecidos por el fundador o los fundadores de la misma, y al ser aplicado este concepto a la sociedad choca con el error teórico de pretender organizarla desde arriba, siendo imposible debido a que los dirigentes sencillamente no pueden recopilar la información necesaria porque en gran parte es práctica, tácita, subjetiva, se encuentra dispersa entre todos los sujetos y cambia continuamente al ser descubierta y transmitida mediante la interacción humana. ${ }^{2}$

Las leyes sobre las que surge el orden espontáneo son descubiertas a lo largo del tiempo, entendido este en un sentido institucional, es decir, no praxeológicamente ${ }^{3}$ - relacionado con la acción humana-, sino tras generaciones y generaciones, siglos y siglos. Inicialmente aparecen como costumbres, modos de actuar repetidos que se van transmitiendo de padres a hijos y a lo largo del tiempo se modifican y depuran, constituyendo la tradición. De esta forma, en las normas generales, abstractas y evolutivas de los órdenes espontáneos se acumula un inmenso volumen de información fruto de la experiencia adquirida por todas las personas que nos han precedido, que cada nuevo miembro de la sociedad recibe y aprende a lo largo de su vida.

En este proceso de descubrimiento de las leyes de los órdenes espontáneos iniciado involuntariamente, han surgido de abajo hacia arriba, consuetudinaria y evolutivamente a lo largo de extensos periodos de tiempo las instituciones sociales como un conjunto de comportamientos pautados libremente asumidos que han resultado ser más adecuados para la pacífica cooperación y

entre los conceptos de orden y organización, orden espontáneo y orden deliberadamente creado.

2 Huerta de Soto (2010: 52-67).

${ }^{3}$ Sobre la diferencia entre el concepto institucional y praxeológico del tiempo, Martínez Meseguer (2012: 163-174). 
prosperidad social. En muchos casos los propios sujetos que actúan conforme a estas leyes no son conscientes de su existencia o desconocen su contenido, pero simultáneamente les resultan sumamente útiles no solo a ellos, sino también a todos los demás miembros de la sociedad porque les proporcionan una información muy valiosa referida a las acciones favorables al desarrollo de la vida en sociedad y a la reacción esperable de los otros, es decir, constituyen una guía del actuar porque permiten prever las consecuencias sociales de la propia actuación y de la actuación de los demás. ${ }^{4}$

En el momento en que se intenta aplicar a la sociedad o a alguna de sus instituciones - la familia, la moral, el derecho, el mercado, el dinero, el lenguaje...- el esquema de organización diseñada de una determinada forma por sus dirigentes, inevitablemente se coarta la libertad individual, lo que impide el libre desarrollo de las relaciones interpersonales y bloquea el proceso de creación y transmisión del conocimiento en la sociedad, estancando el progreso de la civilización.

\section{Eficiencia dinámica y moral}

La eficiencia social, entendida como la capacidad de la sociedad para impulsar la creatividad de las personas y la espontánea coordinación entre ellas, requiere no solo un marco ético mínimo compartido por todos y respaldado por el Derecho - a saber, respeto a la vida, propiedad privada y cumplimiento de los contratos- sino también una auténtica vida moral de sus miembros. ${ }^{5}$

\footnotetext{
4 «Ese es precisamente el papel desempeñado por la tradición y por las instituciones en la sociedad: dar estabilidad y seguridad a la acción humana en su proyección hacia un futuro en esencia incierto», Martínez Meseguer (2012: 136).

5 De acuerdo con Huerta de Soto (2004: 26-59). En el ámbito de la moral, se refiere a unos principios morales que guíen la acción humana.

Sobre la importancia de la moral para la vida social en libertad, Hayek (2008: 95) afirma: «La libertad no ha funcionado nunca sin la existencia de hondas creencias morales».

Por su parte, Novak (1996: 115) defiende que los negocios dependen de la moralidad: «Business has a vested interest in virtue. It cannot go forward with realism, courage, wisdom, honesty, and integrity without a highly motivated and virtuous
} 
La persona es capaz de reconocer el bien y llevarlo a cabo, de este modo en la experiencia moral se orienta el propio actuar hacia la búsqueda y realización del bien, desarrollándose la acción virtuosa. ${ }^{6}$ La moral está inserta en la naturaleza humana, pero no ha sido completamente conocida desde el origen del género humano, sino que se ha ido reconociendo, desarrollando y concretando con el paso del tiempo.

El acervo moral de la sociedad se transmite a lo largo del tiempo por medio de determinadas instituciones sociales - como la familia-que facilitan que en las personas se despierte la experiencia moral y así puedan descubrir y realizar el bien. Cada cual es libre de actuar moralmente, a través del oportuno proceso de aprendizaje e integración en la vida de la moral como guía del proceder, o bien de rechazarla y actuar en sentido opuesto. Esta disyuntiva es condición indispensable de la acción humana ya que sin la facultad de obrar de una forma u otra el comportamiento no sería deliberado y por ende no habría acción humana como tal. ${ }^{7}$

Para que una persona actúe moralmente es preciso que tenga la capacidad de decidir y obrar de un modo u otro, es decir, que actúe libremente. ${ }^{8}$ La moral requiere de este espacio de la libertad en el que en cada acción la persona elige el fin que persigue

work community. It cannot endure without leaders and colleagues in whom many key virtues are internalized. In this and in many other ways, business is dependent on the moral and cultural institutions of the free society: families especially, schools and public civil life».

6 «Without virtue, self government is impossible. The four classic virtues require one to be prudent, just, brave and temperate. [...] Prudence, or wisdom, is seeking the good and the true [...] Justice is ultimately relational. It is the idea that each person is to be given his or her due. [...] Fortitude, or courage, exists when first we are afraid, vulnerable if you will, for then, even then, we will seek to do the good despite the pain, the ridicule, or the attractiveness of the temptation to do wrong. Temperance refers to maintaining or directing the harmony of the whole person. It focuses on realizing within ourselves a peace, a well-ordered soul. This is a supreme challenge», Durant (1996: 7).

7 «La acción humana es una conducta consciente, movilizada voluntad transformada en actuación, que pretende alcanzar precisos fines y objetivos», Mises (2011: 15).

8 Se trata de «la necesidad de una intervención del sujeto agente para que se pueda hablar de una experiencia moral. Esta no sucede con un sujeto meramente pasivo, sino que tiene que «ponerse» en su acción mediante la libertad», Melina (2007: 137). 
y los medios que emplea para ello. Solo en un entorno de libertad es posible desarrollar la innata creatividad humana necesaria para descubrir y realizar el bien a través de acciones concretas en las particulares circunstancias de cada momento. Además el libre ejercicio de la acción humana permite el progreso de la sociedad en la profundización de la moral, que se transmite y asume personalmente de generación en generación.

Este actuar moralmente, es decir, hacer el bien, afecta a otros por cuanto la persona está naturalmente inmersa en un complejísimo proceso de interacciones humanas que conocemos con el nombre de sociedad. ${ }^{9}$ En este dinamismo de relaciones interpersonales la acción moralmente buena reconoce al otro y permite comunicarle un bien. Toda vez que el otro acepte recibir ese bien que se le ofrece, ambas personas habrán coordinado su comportamiento libre y pacíficamente por medio del bien compartido. De esta forma, la moral resulta dinámicamente eficiente puesto que guía hacia la construcción creativa de acciones excelentes, ${ }^{10}$ lo que produce efectos de coordinación social.

Este ajuste mutuo anima tanto a las personas implicadas como a quienes ven o perciben de algún modo ese bien que se comparte, a construir acciones moralmente buenas y así vivir relaciones armoniosas con los demás. A través de las acciones virtuosas podríamos decir que el bien «se expande», de modo que el sujeto que realiza el bien no solo progresa moralmente y coordina su

9 Entendida como «un proceso (es decir, una estructura dinámica) de tipo espontáneo, es decir, no diseñado conscientemente por nadie; muy complejo, pues está constituido por millones y millones de personas con una infinita variedad de objetivos, gustos, valoraciones y conocimientos prácticos, todos ellos en continuo cambio; de interacciones humanas (que básicamente son relaciones de intercambio que a menudo se plasman en precios monetarios y siempre se efectúan según unas normas, hábitos, o pautas de conducta); movidas e impulsadas todas ellas por la fuerza de la función empresarial, que constantemente crea, descubre y transmite información o conocimiento, ajustando y coordinando de forma competitiva los planes contradictorios de los seres humanos, y haciendo posible la vida en común de todos ellos con un número y una complejidad y riqueza de matices y elementos cada vez mayores», Huerta de Soto (2000: 48).

10 «El papel fundamental de las virtudes es entonces integrar los dinamismos de la persona para dirigirlos hacia la acción excelente», Melina (2007: p. 454).Y sobre la estructura del obrar excelente, Melina (2007: 705-760). 
comportamiento con el de aquellos con quienes se relaciona, sino que a la vez impulsa el desarrollo de la capacidad creativa de los demás en su experiencia moral, realizando acciones excelentes, con el consiguiente efecto de coordinación social libre y pacífica.

\section{Moral sexual, creatividad y coordinación}

La experiencia moral abarca de forma particular la vivencia de la afectividad ${ }^{11} \mathrm{y}$ sexualidad, que toca el espacio más íntimo de la persona y tiene consecuencias sumamente importantes para toda la sociedad, ya que influye en la forma de comprenderse a sí mismo, el establecimiento de nuevas familias y la generación de nuevos seres humanos. Se trata del modo de entender el propio ser corporal y la intimidad, de reconocer, interpretar e integrar los sentimientos, emociones y pasiones, y de vivir las relaciones interpersonales ${ }^{12}$ para buscar y hacer el bien y así lograr una vida feliz.

La moral sexual guía a la persona en el reconocimiento del otro y la comunicación interpersonal del bien que genera la unión. Para ello fomenta el desarrollo de la innata creatividad humana en la comunicación afectiva con los demás, esto es, en la integración de los afectos a través de acciones en las que se comparte un bien con el otro. ${ }^{13}$ De esta forma, en las acciones virtuosas el afecto que mueve al sujeto es dirigido al encuentro con el otro a través del bien que se ofrece y comparte. La moral sexual, por lo tanto, favorece espontáneamente la coordinación interpersonal

11 Sobre las cinco dimensiones de la afectividad, a saber, física, psicológica, conductual, cognitiva y asertiva, Rojas (2008: 51-59).

12 «La persona capta al otro en tanto otro, en tanto un tú, no una cosa siendo, esa captación no total, pero sí inmediata porque no requiere demostración: es el yo del otro que se manifiesta como evidente a través de su cuerpo que manifiesta sus potencialidades espirituales propias, mediante el hablar, el comprender, el amar, el mirar, el sonreír, el escuchar, el abrazar, el gesticular», Zanotti (2011: 47). Sobre la corporeidad e intersubjetividad, Zanotti (2011: 46-48).

13 Sobre la llamada «cultura del don» en el capitalismo en general, Felice (2005: 191-194). 
en un ambiente de libertad y concordia ${ }^{14}$ mediante este continuo dar y recibir bienes en el caminar con el otro.

Para lograr llegar a la otra persona, conocerla y dejarse conocer, aceptarla y ser aceptado, comprenderla y ser comprendido, es preciso buscar creativamente nuevas formas de manifestarse, de expresar los afectos, deseos, ideas, expectativas, esperanzas..., de acceder al mundo afectivo del otro y de comunicarse con él. En esta búsqueda continua de la comunicación creativa las personas se expresan a través de diferentes «lenguajes», que podrían diferenciarse entre el lenguaje verbal, los detalles materiales, la servicialidad, la dedicación de tiempo en exclusiva a la otra persona y el contacto físico. ${ }^{15}$

El desarrollo de la comunicación afectiva según la moral en un entorno de libertad acerca a las personas, al permitir que cada uno descubra y emplee los medios que considere más adecuados para relacionarse con los otros. De este modo surge espontáneamente un proceso dinámico en el que las personas se comunican y al profundizar en el conocimiento mutuo son capaces de percibir cada vez más y mejor cómo se encuentra el otro, qué necesita, qué le alegraría, etc. Y por la propia lógica de la relación afectiva, las personas tienden a abrirse y actuar hacia la unión que generan los bienes compartidos, satisfaciendo las necesidades más personales del otro, tales como la de ser escuchado, sentirse comprendido, respetado, valorado y admirado... en una palabra, el anhelo de ser amado.

En la comunicación afectiva con los demás las acciones del sujeto pueden resultar erradas, en la medida en que pretendiendo acercarse al otro y compartir un bien, de hecho la acción no tenga el efecto de favorecer la unión y coordinar a ambas personas, sino que por el contrario dañe su relación, teniendo consecuencias descoordinadoras. Pero la innata capacidad creativa propia del ser humano siempre estará preparada para descubrir una oportunidad de crecimiento en la relación con el otro, de conocimiento mutuo, de apertura y revelación de uno mismo, de dar

14 Sobre la concordia como fruto de la comunicación afectiva, Noriega (2007: 124125).

15 Chapman (2011). 
más de sí mismo y estar dispuesto a acoger más del otro. Cada momento -y quizá especialmente tras una acción equivocada en la relación con el otro- brinda la posibilidad de acercarse a él, reconocer cómo está y darle aquello que necesita.

A lo largo de este recorrido personal de relación con el otro, en el proceso de crecimiento y maduración en la unión afectiva a través de acciones creativas que dirigen el afecto a la comunicación de un bien al otro, al mismo tiempo que se ajustan los comportamientos individuales cuando el otro acoge el bien que le es ofrecido, surge dinámicamente la novedad del «nosotros». La comunicación afectiva de dos personas orientada por la moral da el fruto de la aparición de un nuevo «nosotros» a partir de los dos «yoes» de los sujetos que actúan. Esta unión interpersonal que nace de la comunicación afectiva se desenvuelve poco a poco al ir dando forma a espacios comunes, descubriendo afectos y deseos compartidos, preparando proyectos en común, etc.

Este «nosotros» se concreta creativamente en cursos de acción elegidos y realizados en común, no como una fusión indiferenciada de las personas implicadas, ni como una reducción a la nada de una de ellas que resulta dominada por la otra, sino en una auténtica unión interpersonal en continuo ajuste y expansión en la que ambos ofrecen y acogen, dan y reciben los bienes que comparten. ${ }^{16} \mathrm{El}$ dinamismo del encuentro interpersonal en la comunicación afectiva es altamente coordinador, pues a la iniciativa individual de cada uno se añade el impulso de las acciones decididas y llevadas a cabo conjuntamente persiguiendo un fin común, abriéndose paso así a la cooperación entre estas personas.

16 «La amistad implica dos dimensiones intrínsecas con un influjo decisivo en la actuación: por un lado, la mutua unión, gracias a la cual es posible una transformación mutua y una concordia singular que permite identificar cuál es el bien que está en juego y verlo como relevante. Por otro lado, la alteridad y distinción, necesaria en toda amistad, que abre un espacio a la justicia, por lo que nos permite dirigir el bien descubierto hacia la otra persona», Noriega (2007: 125). 


\section{Matrimonio y eficiencia dinámica}

La mayor unión interpersonal es aquella en la que no solo se comparten determinados bienes, sino que se entregan y acogen recíprocamente ambas personas. ${ }^{17}$ Esta unión es incomparable a cualquier otra porque su unicidad reside en que el bien que se comparte es la propia vida, el pasado, el presente y el incierto futuro. ${ }^{18}$ Ya no se trata solo de dar y recibir bienes, sino de darse y recibirse mutuamente de por vida, trabajando diariamente en la construcción creativa del hogar común. De esta forma, el hombre y la mujer concretan su libertad en la opción fundamental del compromiso recíproco del don de sí, que exige una perseverante disposición a la cooperación, plasmada activamente en un proceso de ajuste y adaptación sin límites. ${ }^{19}$ Esta paradigmática unión interpersonal del hombre y la mujer constituye el fundamento de la institución del matrimonio y la familia. ${ }^{20}$

Los esposos se introducen así en una dinámica en la que tienen por delante un inmenso horizonte de posibilidades por descubrir y construir juntos, pues cuanto más se conozcan y más íntima y de mayor calidad sea su comunicación, mayor será su capacidad de percibir las necesidades del otro y actuar creativamente para satisfacerlas. Esta específica unión interpersonal — que ha sido denominada «comunión de personas»—, requiere el ejercicio de

17 «A successful marriage requires the complete gift of the self to the other person. It is not reasonable to give of the self at the same level unless there is a complete commitment. These are the key elements of marriage: commitment and self-giving to another person", Roback Morse (2008: 73).

18 Sobre la diferencia entre riesgo e incertidumbre en el matrimonio, Roback Morse (2008: 76-81).

19 Roback Morse (2008: 37) sostiene que en comparación con el mercado, «the family is an even more fundamental example of an undirected social order that arises spontaneously from the needs and activities of its participants».

${ }^{20}$ Roback Morse (2008: 90) defiende que «the marriage relationship is at the core of the family and the basis upon which the kinship network is built. Marriage is not a mere contract and is not held together by contract. Marriage is held together by love. Through the love between them, the married couple binds the other generations to themselves and to each other. That is why this book about libertarian political theory and free market economics needs to talk about love».

Sobre el matrimonio como institución presente en todas las sociedades, Davis (1985) 
la creatividad humana para profundizar en el don y acogida mutua, haciendo al otro cada vez más partícipe en la propia vida, al mismo tiempo que es estímulo para su desarrollo a través de nuevas acciones creativas.

El don de sí implica la unión total del hombre y la mujer, expresada a través de su corporalidad en la relación sexual. ${ }^{21} \mathrm{La}$ sexualidad llama a la comunicación con el otro y necesita de él para expresarse en plenitud ${ }^{22}$ invita a salir de uno mismo para ir al encuentro del otro, y es justamente en la unión con él donde puede vivirse en totalidad. ${ }^{23}$ Para que la unión sexual no sea mera conjunción de cuerpos, sino auténtico encuentro interpersonal, ${ }^{24}$ es necesario poner en práctica la creatividad, comunicándose afectivamente e integrando todos los dinamismos de la persona hacia el bien de la unión con el otro. El impulso sexual orientado por la moral es esencialmente coordinador, puesto que se trata de la tendencia humana de mayor carácter social. ${ }^{25}$

En la cooperación con el otro en el desarrollo de la comunión de personas se da una suerte de sinergia, de tal forma que de la acción de las dos personas surge algo mayor que la suma de sus acciones individuales, se crea algo que les sobrepasa. La formación de una familia expande ese nuevo «nosotros» y tiende a ser

21 «El bien inmanente del copular se encuentra en la plenitud humana que comporta entregarse y acogerse recíprocamente en totalidad, generando una comunión de personas abierta a acoger una nueva vida. La intensidad del placer que conlleva esta acción es precisamente signo de la plenitud humana que comporta», Noriega (2012: 48).

22 «A diferencia de otras funciones biológicas, la función sexual humana goza de un carácter absolutamente peculiar: necesita de otro para poderse expresar cabalmente», D’Agostino (2006: 196).

23 «El ser humano es creado hombre y mujer, en comunión de uno con el otro; en esa comunión matrimonial tiene plenas relaciones sexuales y es llamado a trabajar y a poblar la tierra», Zanotti (2011: 36).

24 «El encuentro sexual entre el hombre y la mujer comporta una novedad con respecto a las demás especies animales: es un encuentro cara a cara [...] Se inicia a través de los actos de ternura, con sus caricias, sus palabras, sus besos y sus silencios, gracias a los cuales el cuerpo de ambos, con ritmos diferentes, se van preparando para poder interactuar sexualmente», Noriega (2012: 44).

25 «De todos los impulsos, inclinaciones y tendencias "naturales" del ser humano, el deseo sexual fue y sigue siendo el más irrefutable, obvia y unívocamente social», Bauman (2011: 59).

También Roback Morse (2005: 48-63). 
fuente de creatividad, puesto que el hombre y la mujer construyen su nuevo día a día de la vida en común, trabajan su propio estilo familiar de comunicación interpersonal, relaciones familiares, sociales y laborales, organización de la tareas domésticas, profesionales y del tiempo libre, etc.

A través de la comunicación en la familia se descubren y desarrollan las relaciones de complementariedad entre las personas, entre el hombre y la mujer, los hermanos, y los miembros de diferentes generaciones. Inconscientemente se integra la complementariedad en la vida como positiva para la comunicación con los otros, ya que favorece el trabajo en común y la ayuda mutua, coordina los comportamientos individuales y permite que cada uno logre más fines que si actuara aisladamente.

Desde su inicio la familia contiene la novedad de las relaciones a las que da origen la unión de vida del hombre y la mujer. En primer lugar, la esponsalidad, y posteriormente en muchos casos paternidad y maternidad, filiación y fraternidad. Estas nuevas relaciones brindan a las personas implicadas la oportunidad de vivir la experiencia moral a través de la realización de acciones excelentes y de este modo crecer en la armoniosa coordinación familiar.

El culmen de la creatividad humana y familiar es, sin duda alguna, la procreación, la capacidad de engendrar vida y acoger a los hijos. ${ }^{26} \mathrm{El}$ encuentro sexual del hombre y la mujer puede dar lugar al inicio de una nueva vida humana, una creación absolutamente distinta a la realidad anterior del padre y de la madre, que supera infinitamente las aportaciones personales que ambos han realizado. El desarrollo de este nuevo ser humano en el seno materno, surgido de la unión de sus padres, es como una explosión de creatividad que sigue su curso propio sin que ellos vuelvan a intervenir, llegando incluso en el futuro a poder desarrollar su innata y personal capacidad creativa.

26 «Sin miedo a exagerar podemos afirmar que uno de los momentos en que se expresa con más potencia la creatividad del ser humano es en la experiencia de la paternidad», Vives (2011: 190). 


\section{II \\ EFICIENCIA DINÁMICA Y EDUCACIÓN AFECTIVO-SEXUAL}

\section{Educación afectivo-sexual familiar y eficiencia dinámica}

$\mathrm{Al}$ aumentar la familia con la llegada de los hijos, los padres se enfrentan al reto de su educación, una oportunidad única de acompañar personalmente a sus hijos en su proceso de maduración afectivo, intelectual, físico, social y espiritual, que los padres podrán vivir desarrollando al máximo su potencial creativo para comunicarse con ellos y motivarles para que avancen en su recorrido educativo. La educación en el ámbito de la moral sexual requiere que los padres actúen creativamente, percibiendo los cambios y las necesidades de cada uno de sus hijos a lo largo de su crecimiento y adaptándose a ellos para abrir espacios de comunicación en los que acompañarles en el descubrimiento de su corporeidad e intimidad y ayudarles a integrar su afectividad.

Allí donde nacen y crecen los niños rodeados de sus padres, hermanos y demás familiares, es donde naturalmente se transmite y aprende la búsqueda, el reconocimiento y la realización del bien. ${ }^{27}$ De este modo los niños pueden integrar de forma natural su sexualidad, aprender a comunicarse con los otros, a expresarse afectivamente y comprender el lenguaje de los demás, a percibir cómo se encuentran y qué necesitan. Es en este entorno familiar donde los niños y adolescentes viven la experiencia moral, descubriendo el sentido y significado de su sexualidad, hasta poder llegar a comprender la grandeza y unicidad de la unión interpersonal del hombre y la mujer fruto del don total de sí en la institución del matrimonio.

En la familia, al ser naturalmente el núcleo y primer entorno de relaciones interpersonales, así como la unidad más fundamental

27 «The loving family is surely the foundation of the moral and cultural leg of a free society, just as property rights and contract law are the foundations of the economic leg, and constitutionally limited government and freely elected rulers are of the political leg. Loving families are just as essential to a free society as property rights and a constitution», Roback Morse (2008: 266). 
de cooperación social, ${ }^{28}$ los niños aprenden desde pequeños a vivir en sociedad y a ajustar su actuación a las necesidades de los demás. Gracias a la relación con sus hermanos o primos descubren que no son los únicos que tienen necesidades y requieren la atención de los padres, sino que existen otros que están en una situación similar a la suya. También toman conciencia poco a poco de las consecuencias que sus actos tienen en los demás y diferencian los comportamientos que favorecen el acercamiento y la ayuda mutua de los que crean enfrentamientos y discordias. Asimismo, los niños aprenden a compartir bienes y actuar coordinadamente con los demás miembros de la familia, uniendo sus esfuerzos y poniendo los medios para que el desarrollo de las relaciones familiares sea lo más profundo y armonioso posible. ${ }^{29}$

La realización de la experiencia moral por el hijo adolescente implica el ejercicio de su libertad al apreciar el bien de lo que sus padres le transmiten e incorporarlo dinámicamente a su vida como guía de su actuar. Solo así es posible que los adolescentes actúen moralmente y desarrollen su innata creatividad en la concreción de acciones virtuosas. Vivir una vida moralmente buena en el ámbito de la sexualidad requiere poner en movimiento la capacidad creativa personal para construir relaciones de auténtico encuentro y comunicación interpersonal.

La tarea educativa familiar implica personalmente tanto a los padres como a los hijos, en la medida en que exige el desarrollo de sus mejores capacidades comunicativas para trabajar la unión interpersonal mediante la incesante realización de acciones en las que se ofrezcan y reciban bienes. De esta forma, el proceso de educación afectivo-sexual impulsa espontáneamente la coordinación interpersonal al promover un continuo aprendizaje y profundización en la comunicación afectiva mediante acciones orientadas a compartir bienes y crear ámbitos de unión. La educación afectivo-sexual en la familia, al transmitir la moral sexual, resul-

28 «Just as the market is a system of social cooperation, so too is marriage the most basic and fundamental unit of social cooperation», Roback Morse (2009: 32).

29 Sobre la familia como institución ireemplazable para el desarrollo moral y el bienestar de los niños, así como escuela de cooperación, Roback Morse (2008: 27-57 y 95-185). 
ta dinámicamente eficiente porque tiende a estimular la creatividad tanto de los padres como de los hijos y su resultado consiste en crecer en la unión interpersonal, lo que implica una alta coordinación de sus miembros.

\section{Iniciativas sociales de educación afectivo-sexual}

La transmisión de la moral sexual en la familia no solo promueve la creatividad y coordinación dentro de la propia institución familiar, sino que impulsa también la comunicación afectiva de cada uno de los miembros de la familia con otras personas, y de la familia como ese «nosotros» con otras familias. En este sentido, si los padres consideran oportuno buscar el apoyo de otras personas en el proceso de educación de sus hijos en la afectividad y sexualidad, se reunirán con otras familias, formarán una asociación, acudirán a una iniciativa educativa que ofrezcan otras personas, a una iglesia o institución religiosa,...

Así, la capacidad creativa de los padres desarrollada ante el reto de la educación de sus hijos en la moral sexual puede dar lugar a asociaciones de iniciativa privada que favorezcan la tarea educativa de la familia, aunando esfuerzos y facilitando la coordinación de las personas. Al poner en común voluntariamente el trabajo y la creatividad de diferentes familias que persiguen fines comunes, en estas agrupaciones de la sociedad civil se desempeña una importante actividad de cooperación interpersonal, tendiendo a aumentar la eficiencia dinámica de la sociedad en su conjunto.

Por otro lado, el proceso de transmisión de la moral sexual de padres a hijos, la mutua colaboración entre familias a través de las iniciativas sociales para mejorar la comunicación afectiva y crecer armónicamente en la unión familiar, así como la asunción libre y personal de la búsqueda del bien por los adolescentes, permiten el continuo desarrollo espontáneo y evolutivo de la propia moral sexual. Con el transcurso del tiempo y el libre ejercicio de la acción humana creativa en la comunicación afectiva, aumenta el inmenso volumen de información sintetizado en la moral sexual y continúa su proceso de descubrimiento, desarrollo 
y compleción, así como su concreción ante los nuevos desafíos de cada momento.

Para que la transmisión de la moral pueda llevarse a cabo, es necesario que el espacio familiar donde los padres comunican creativamente esta experiencia a sus hijos sea respetado y se mantenga libre del control de terceros y de injerencias por parte del estado. Los padres no solo son naturalmente los primeros responsables de la educación de sus hijos, sino que además son quienes pueden tener el mayor conocimiento acerca de su personalidad, desarrollo y necesidades, así como de la situación familiar concreta en cada momento, para decidir qué es lo que más les conviene, cómo comunicarse con ellos y educarles en la afectividad y sexualidad.

Es más, la salvaguarda de la capacidad creativa de padres e hijos en la vivencia e interpretación de la experiencia moral, así como de la cooperación social a la que da lugar, exige el respeto a la libertad de educación de los padres muy especialmente en el ámbito de la afectividad y sexualidad. Para no obstaculizar el progreso en las relaciones familiares y de este modo poder extenderse creativamente la ayuda mutua y la consecuente coordinación familiar y social, resulta crucial que tanto el poder político como terceras personas $\mathrm{u}$ organizaciones respeten la libertad de educación de los padres, así como de las asociaciones en las que confíen como apoyo a su tarea educativa.

La sociedad y sus instituciones — concretamente, la moralno solo han surgido y se desenvuelven históricamente como órdenes espontáneos, sino que únicamente respetando este orden es posible desarrollar la innata creatividad humana en la consecución de los fines individuales a través de los medios que cada cual juzgue más apropiados, lo que tiende a la coordinación social, que da lugar al llamado «big bang social», ${ }^{30}$ esto es, a una expansión del progreso social tan amplia y armoniosa como sea humanamente factible en cada momento y lugar.

30 Huerta de Soto (2000: 47). 


\section{Educación afectivo-sexual pública e ineficiencia social}

Por el contrario, cuando el estado establece la obligatoriedad de la enseñanza en afectividad y sexualidad está violando la libertad de educación de los padres porque da preeminencia al poder político sobre ellos, al ser él quien fija en régimen de monopolio qué comportamientos y actitudes se enseña a los niños y adolescentes sobre la sexualidad. ${ }^{31}$ De esta forma, el estado se erige en autoridad moral y entra en la esfera más privada de la vida de los menores, conminándoles a entender su corporeidad, interpretar sus emociones, sentimientos y pasiones, formarse un juicio sobre la procreación y buscar relacionarse con los demás en un sentido elegido con antelación por el poder político.

La educación afectivo-sexual pública impone unas normas de comportamiento moral que son determinadas por el gobierno de turno, impidiendo así la libre aceptación e integración de las mismas en la vida. De esta forma, deviene imposible que el sujeto actúe moralmente, porque al no tener la posibilidad de aceptar la moral o rechazarla y actuar en sentido opuesto, se restringe su capacidad de acción, limitándose a seguir las normas morales por obligación, miedo o incluso servilismo, con la consiguiente desintegración afectiva. La moral necesita el espacio de la libertad en el que la persona que actúe siguiendo sus pautas lo haga porque libremente quiere asumirlas.

Tan pronto como se obligue a cumplir unas determinadas pautas morales a través de la educación afectivo-sexual pública, se obstaculiza el desarrollo de la innata creatividad humana, impidiendo que los padres puedan actuar creativamente para comunicarse con sus hijos y transmitirles dinámicamente la experiencia moral de la mejor forma que puedan según el crecimiento, la personalidad y las circunstancias de cada hijo. ${ }^{32}$ Asimismo, se bloquea la capacidad creativa del hijo para concretar la moral libremente asumida en acciones virtuosas movidas por afectos dirigidos

31 Sobre la educación en general establecida por el estado en régimen de monopolio ver Soriano (2012: 79).

32 Roback Morse (2008: 168-174) compara las deficiencias de la economía planificada con las de la educación planificada. 
a compartir bienes con los otros. El niño simplemente cumple las normas morales que le impone el estado, sin realizar una auténtica experiencia moral.

El intervencionismo estatal en la educación afectivo-sexual es socialmente ineficiente porque dificulta la libre actuación creativa de padres e hijos en el ámbito de la experiencia moral, lo que entorpece la cooperación social fruto de la comunicación interpersonal desarrollada moralmente. También provoca efectos de descoordinación porque quienes establecen qué principios se enseñan, en qué momento y de qué manera carecen del conocimiento de primera mano de los niños que, sin embargo, sí tienen naturalmente los padres. La educación afectivo-sexual estatal no puede adaptarse a las circunstancias personales de cada niño, tales como su historia, situación familiar, madurez, etc., sino que aplica sus objetivos y enseña sus contenidos igualitariamente a todos, con las dañinas consecuencias que esto puede tener para el niño y para la sociedad en su conjunto.

Además, la actividad del estado como agente moralizante que establece e impone los principios que deben guiar la conducta sexual trunca el proceso de desenvolvimiento consuetudinario y evolutivo de la moral sexual en la sociedad. Se paraliza así la espontánea profundización de la moral en la comunicación afectiva y la vivencia de la sexualidad como guía de la acción de las personas hacia el bien. La acumulación de esa inmensa cantidad de información recogida a lo largo de la historia de la humanidad y concretada en la institución de la moral se detiene, porque aunque el estado pretendiera colaborar en el ulterior desarrollo de la misma, ni con el mejor equipo de funcionarios brillantes y capaces sería posible recopilar todo ese conocimiento implícito en la moral proveniente de la experiencia de tantas y tantas personas que nos han precedido.

\section{Educación afectivo-sexual pública y manipulación infantil}

En esta etapa de la vida los adolescentes, en su búsqueda de autoafirmación individual, son fácilmente influenciables por las ideas más imprudentes, a la vez que viven cierta inseguridad por el 
despertar del instinto sexual. ${ }^{33}$ Por eso, la educación que reciben los niños y adolescentes acerca de la interpretación y vivencia de la afectividad y la sexualidad puede tener un tremendo impacto en ellos y predisponerles a comunicarse afectivamente con otras personas y formarse un juicio sobre las relaciones familiares y la procreación en un determinado sentido.

La educación afectivo-sexual pública no es realmente «educación», sino una auténtica «regulación» más del estado, que en este caso interviene para controlar el desarrollo individual, familiar y social de los niños, teniendo así la posibilidad de manipularles en beneficio de los intereses de unos pocos. Quienes reciban educación afectivo-sexual pública serán más fácilmente moldeados al antojo de las mayorías políticas de cada momento, puesto que probablemente se entenderán a sí mismos, interpretarán la procreación y las relaciones con los otros y, en último término, tenderán a actuar en este ámbito tal y como haya previsto el estado.

La posibilidad de manipular de manera tan sencilla a los niños podría animar al gobierno a «jugar» a rediseñar la sociedad como si del trabajo de un «ingeniero social» se tratara. En este sentido, a través de la intervención política en la educación afectivo-sexual de los niños, el estado podría manejar a las personas hasta límites insospechados y, a la larga, llegar a destruir las instituciones sociales fundamentales del matrimonio y la familia, y experimentar produciendo de la nada e imponiendo a los niños nuevos modelos de personalidad y de relaciones con los otros. ${ }^{34}$

Al controlar la educación afectivo-sexual, el estado se extralimita en sus funciones de forma flagrante, porque viola la libertad de educación de los padres y se entromete en las más íntimas esferas de la vida de los niños, lo que cercena su libertad, limita su capacidad creativa, impide que puedan actuar moralmente y, por ende, dificulta el proceso espontáneo de coordinación social, al mismo tiempo que bloquea el desarrollo y perfecciona-

33 Guardini (43-46).

34 Nogales Lozano (2012: 183) sostiene que la coacción educativa va acompañada de «una perversa tarea de reingeniería social sobre la propia institución familiar». 
miento evolutivo de la moral en el ámbito de la afectividad y sexualidad.

\section{III \\ INTERVENCIONISMO ACTUAL EN EDUCACIÓN AFECTIVO-SEXUAL}

\section{Control público de la educación afectivo-sexual}

Actualmente en España nos encontramos ante un creciente intervencionismo del poder político en la educación afectivo-sexual. La ley de salud sexual y reproductiva de 2010 - más conocida como «ley del aborto»— comienza estableciendo políticas públicas de salud sexual y reproductiva, entre las que destaca la incorporación al sistema educativo de «educación afectivo sexual y reproductiva» con el objetivo de lograr «la convivencia y el respeto a las opciones sexuales individuales», así como «la corresponsabilidad en las conductas sexuales, cualquiera que sea la orientación sexual». .35

El texto legal no define en qué consiste la educación afectivo sexual y reproductiva a la que se refiere, pero en sus disposiciones generales hace referencia a expresiones imprecisas como «vida sexual segura» en "un entorno libre de coerción, discriminación y violencia», incluyendo el ejercicio de «la libertad de tener hijos y de decidir cuándo tenerlos»... ${ }^{36}$

¿Cómo es una «vida sexual segura»? En un primer momento puede parecer que se trata de aquélla en la que todo contacto sexual es libremente aceptado o realizado, sin que medie presión ni violencia alguna. Pero si profundizamos en la cuestión, quizá abarque también el hecho de poder tener relaciones sexuales sin dificultades físicas, psicológicas o afectivas, o incluso que esas relaciones sean plenamente satisfactorias para las personas que las viven. En este caso la educación para una vida sexual segura

35 LO 2/2010, de 3 de marzo, de salud sexual y reproductiva y de la interrupción voluntaria del embarazo, art. 5.1.a. y 5.2.

36 Ídem, arts. 3.1. y 2.b y c. 
establecería los fines que los alumnos deberían buscar en las relaciones sexuales, fijados por la mayoría política de turno.

¿Y cuál es el significado de lo que la ley llama «sexo seguro»?37 ¿Tener relaciones sexuales con la seguridad de que no se contraerá una enfermedad de transmisión sexual?, ¿la seguridad de no sufrir problemas psicológicos posteriores derivados de la experiencia sexual?, ¿de que no haya consecuencias ni efectos secundarios?, ¿quizá de que en caso de embarazo la madre tenga la seguridad de que el padre también se responsabilizará de la nueva criatura?...

Por otro lado, ¿en qué consiste la «no discriminación»? ¿Será la imposición a los niños de un igualitarismo sexual que les haga pensar en la igual valoración moral de las relaciones sexuales que impliquen la entrega y acogida recíproca de por vida del hombre y la mujer, de aquellas en las que se usa al otro para obtener la máxima sensación de placer, o de los contactos sexuales entre dos personas del mismo sexo, o entre tres o más personas, o entre personas y animales, o personas y objetos...?

¿Y qué comprende «la libertad de tener hijos y de decidir cuándo tenerlos»? En este caso la ley tampoco especifica exactamente lo que quiere decir, pero en su contexto general parece que considera el aborto - al que está dedicada la segunda parte de la ley- un derecho mediante el cual ejercer esta libertad terminando con la nueva vida existente en el interior de la mujer cuando no se quiera tener un hijo en ese momento. Sea como fuere, la educación afectivo-sexual y reproductiva introducida en esta ley constituye un paradigmático intento de controlar a los niños y adolescentes en el ámbito de la moral sexual. Pero desgraciadamente esta pretensión no es nueva.

El objetivo de controlar la educación afectivo-sexual por parte del estado ha sido claramente expresado en la ley que estamos comentando, pero unos años antes se había regulado ya la enseñanza obligatoria de algunas actitudes y comportamientos relativos a la afectividad y la sexualidad. Así, la ley de educación de 2006 estableció como uno de los fines de la educación el «reconocimiento

37 Ídem, art. 5.1.f. 
de la diversidad afectivo-sexual, así como la valoración crítica de las desigualdades, que permita superar los comportamientos sexistas». ${ }^{38} \mathrm{Y}$ entre sus objetivos aparecía desarrollar una «actitud contraria a los estereotipos sexistas» $\mathrm{y}$ "conocer y valorar la dimensión humana de la sexualidad en toda su diversidad».39

¿Cuál es la «diversidad afectivo-sexual» de la que habla la ley de educación? ¿Será la posibilidad de comunicarse afectivamente y tener contacto sexual tanto con personas del otro sexo como del mismo, con varias a la vez o con animales u objetos? ¿Y qué son los inaceptables "comportamientos y estereotipos sexistas»? ¿Comprenderá esta expresión algunas pautas morales transmitidas de generación en generación tales como la monogamia, la fidelidad matrimonial, la especial dedicación de la madre al recién nacido durante la lactancia...?

\section{Prevención de ETS, embarazos inesperados y abortos}

Esta regulación de la educación afectivo-sexual se justifica por la necesidad de "prevenir, especialmente en personas jóvenes, las infecciones de transmisión sexual, los embarazos no deseados y los abortos», así como "establecer las condiciones para que la decisión de tener hijos y cuándo tenerlos se adopte de forma libre y responsable». ${ }^{40}$ La educación afectivo-sexual pública, por lo tanto, tiene contenidos relacionados con la prevención de las ETS y los embarazos inesperados, que podrían enseñarse desde la estrategia $\mathrm{ABC}$ (abstinence, be faithful, use a condom) o centrándose exclusivamente en la anticoncepción, extremo que la ley deja abierto, pero al realizar una lectura general de la misma parece que considera únicamente la anticoncepción como medio para regular la procreación.

Respecto del aborto, curiosamente la ley por un lado diseña un «derecho a la prestación sanitaria de la interrupción voluntaria

${ }^{38}$ Ley orgánica 2/2006, de 3 de mayo, de educación, preámbulo.

39 Ídem, art. 17.m y 23.k.

$40 \mathrm{LO} 2 / 2010$, de 3 de marzo, de salud sexual y reproductiva y de la interrupción voluntaria del embarazo, preámbulo. 
del embarazo» ${ }^{41}$ y por otro, dice pretender prevenir los abortos. Simultáneamente establece el aborto como un nuevo derecho y juzga necesario prevenir su ejercicio. Si se considera un derecho a enseñar a los adolescentes mediante la educación afectivo-sexual pública, ¿por qué se intenta a la vez limitar su realización?

\section{Educación afectivo-sexual y educación para la ciudadanía}

La intervención pública en la educación afectivo-sexual se realiza a través de materias incorporadas al sistema educativo. Si bien es cierto que la ley de salud sexual y reproductiva anuncia que se añadirá específicamente «la formación en salud sexual y reproductiva», ${ }^{42}$ la actual ley de educación ya había introducido la famosa «educación para la ciudadanía y los derechos humanos», 43 con la ambición de hacer que los alumnos profundizaran en los principios morales personales, incluyendo para ello determinados contenidos de educación afectiva y sexual, entre los que figuraban expresiones no definidas claramente. ${ }^{44}$

Así, por ejemplo, se establecía que uno de los objetivos de esta nueva asignatura era rechazar las «discriminaciones existentes por razón de orientación afectivo-sexual» y que los alumnos aprenderían a valorar críticamente «la división social y sexual del trabajo» y los «prejuicios sociales sexistas y homófobos». En la misma línea, el primer criterio de evaluación de esta asignatura consistía en «identificar y rechazar situaciones de discriminación hacia personas de diferente género u orientación afectivo-sexual». ${ }^{45}$

Tras el cambio de gobierno en España a finales de 2011 se modifica el contenido de educación para la ciudadanía, ${ }^{46}$ eliminando

41 Ídem, art. 18.

42 Ídem, art. 9.

43 Ley orgánica 2/2006, de 3 de mayo, de educación, arts. 18.3 y 24.3.

44 Sobre el adoctrinamiento en esta asignatura, Trillo-Figueroa (2008).

45 Real decreto 1631/2006, de 29 de diciembre, por el que se establecen las enseñanzas mínimas correspondientes a la educación secundaria obligatoria, BOE, 5-01-2007, pp. 718-719.

46 Real decreto 1190/2012, de 3 de agosto, por el que se modifican el real decreto $1513 /$ 2006, de 7 de diciembre, por el que se establecen las enseñanzas mínimas de la educación primaria, y el real decreto 1631/2006, cit. 
las referencias a la sexualidad, pero se mantienen intactas todas las disposiciones educativas generales, así como las que regulan las demás materias, en las que también se habían introducido conceptos imprecisos relacionados con la educación afectivo-sexual. De esta forma, siguen vigentes las capacidades a desarrollar transversalmente en toda la educación secundaria consistentes en «rechazar los comportamientos sexistas» $\mathrm{y}$ "conocer y valorar la dimensión humana de la sexualidad en toda su diversidad», ${ }^{47}$ a las que ya nos hemos referido.

Asimismo, entre los criterios de evaluación de la asignatura de biología y geología de 3.ํㅗ se mantiene el de separar la vivencia de la sexualidad de la procreación a través de los métodos anticonceptivos. ${ }^{48}$ Por otro lado, una de las competencias lingüísticas de secundaria pretende que los alumnos utilicen el lenguaje para «la eliminación de estereotipos y expresiones sexistas», a la vez que la asignatura de matemáticas cuenta con el objetivo de «aplicar las competencias matemáticas adquiridas para analizar y valorar fenómenos sociales como la igualdad de género». ${ }^{49}$

A pesar de la modificación de educación para la ciudadanía, actualmente nos encontramos ante una regulación estatal en materia de educación afectivo-sexual. Pero la intromisión del estado en la moral sexual desgraciadamente llega mucho más allá. En los últimos diez años parece como si el poder político tuviera una obsesión con legislar, controlar y modificar la comunicación afectiva y las relaciones sexuales de las personas, pues se ha ido aprobando toda una colección de leyes que regulan las relaciones afectivas y familiares, se entrometen en la sexualidad e

47 Ley orgánica 2/2006, de 3 de mayo, de educación, art. 23.d) y k).

48 «Comprender el funcionamiento de los métodos de control de la natalidad y valorar el uso de métodos de prevención de enfermedades de transmisión sexual. A través de este criterio se intenta comprobar si los alumnos y las alumnas distinguen el proceso de reproducción como un mecanismo de perpetuación de la especie, de la sexualidad entendida como una actividad ligada a toda la vida del ser humano y de comunicación afectiva y personal», Real decreto 1631/2006, de 29 de diciembre, por el que se establecen las enseñanzas mínimas correspondientes a la educación secundaria obligatoria, BOE, 5-01-2007, p. 698.

49 Ídem, pp. 686 y 752, respectivamente. 
intimidad de la persona y la familia y rediseñan instituciones surgidas de forma evolutiva y consuetudinaria a lo largo de siglos. ${ }^{50}$

IV

\section{HACIA LA LIBERTAD DE EDUCACIÓN EN AFECTIVIDAD Y SEXUALIDAD}

Ante el panorama descrito no solo de la ineficiente intervención estatal en la educación afectivo-sexual de los niños y adolescentes, sino también del inmenso peligro que tal regulación entraña para el desarrollo personal y social, proponemos emprender el camino hacia el restablecimiento de la libertad de educación de los padres en la afectividad y sexualidad. Así podrá continuar tanto la concreción de la moral ante los retos de cada época, como el proceso espontáneo de cooperación social al que conduce el desarrollo de la innata capacidad creativa en la comunicación afectiva interpersonal guiada por la búsqueda del bien.

En primer lugar, habría que derogar la legislación educativa que afecta a la moral, especialmente en el ámbito de la afectividad y sexualidad y cancelar todos los programas y talleres de educación afectivo-sexual públicos. De esta forma ya no sería el estado quien establecería e impondría a los niños la moral sexual, sino que la experiencia moral en relación con la afectividad y sexualidad se transmitiría y aprendería en la familia y serían únicamente los padres quienes podrían decidir asociarse a otras familias

\footnotetext{
50 Se trata de la Ley orgánica 1/2004, de 28 de diciembre, de medidas de protección integral contra la violencia de género, Ley 13/2005, de 1 de julio, por la que se modifica el código civil en materia de derecho a contraer matrimonio, Ley 15/2005, de 8 de julio, por la que se modifican el código civil y la ley de enjuiciamiento civil en materia de separación y divorcio, Ley 14/2006, de 26 de mayo, sobre técnicas de reproducción humana asistida, Ley 3/2007, de 15 de marzo, reguladora de la rectificación registral de la mención relativa al sexo de las personas, Ley orgánica 3/2007, de 22 de marzo, para la igualdad efectiva de mujeres y hombres, Ley 14/2007, de 3 de julio, de investigación biomédica, Ley orgánica 2/2010, de 3 de marzo, de salud sexual y reproductiva y de la interrupción voluntaria del embarazo, Ley 14/ 2012, de 28 de junio, de no discriminación por motivos de identidad de género y de reconocimiento de los derechos de las personas transexuales.

Sobre la modificación del matrimonio, Martínez-Calcerrada (2005) y D’Agostino (2006: 220-226).
} 
para apoyarse mutuamente en la educación efectivo-sexual de sus hijos, o bien acudir a las iniciativas sociales de educación afectivo-sexual ya existentes.

En este sentido, a día de hoy hay numerosas iniciativas de la sociedad civil que apoyan la tarea de los padres ofreciéndoles una ayuda concreta en la educación afectivo-sexual de sus hijos. Es el caso de los programas Teen STAR, Aprendamos a amar, SABE , Actio answers y StoryBoarding, Protege tu corazón, Saber amar, Aprendiendo a querer, Theology of the Body for Teens, ${ }^{51}$ etc.

En segundo lugar, para terminar con la intervención estatal en educación afectivo-sexual, habría que eliminar todas las subvenciones de las diferentes administraciones públicas concedidas para implementar programas de educación afectiva o sexual. Así solo permanecerán los programas e iniciativas de educación afectivosexual financiados con fondos privados que sean demandados por los padres como apoyo en la tarea educativa de sus hijos.

Caminar hacia la reconquista de la libertad fortaleciendo desde la sociedad civil las instituciones necesarias para la eficiencia dinámica de los procesos sociales - tales como la moral y la familia - es probablemente la mejor forma de favorecer el desarrollo de la innata capacidad creativa del ser humano que conduce a la libre y espontánea coordinación de los comportamientos de los miembros de la sociedad.

\section{REFERENCIAS BIBLIOGRÁFICAS}

Acton, L. (1985): Essays in the history of liberty. Shadeland, IN: Liberty Fund.

Anderson, C. (2006): «The Family Beyond Ideology». Familia et Vita, vol. $11-12$, n. $\mathrm{o}$.

Arendt, H. (2002): Los orígenes del totalitarismo. Madrid: Alianza.

51 www.teenstar.es, www.desarrolloypersona.org/index.php/cursos/programasde-educacion-afectiva-y-sexual, www.ivaf.org/sabe.htm, www.giftandtask.org/GT_ Colegios_ActA.html, www.giftandtask.org/GT_Colegios_STB.html, protegetucorazon. com, http:/ / www.youtube.com/watch?v=dk9bS-eeh1E, http: / / allianceforfamily. org/our-projects/?lang=es, thetheologyofthebody.com/information/teens, respectivamente (consultados 10-10-2013). 
Bargueño, J.R. y Gil Rodríguez de Clara, V.E. (ed.) (2011): La familia como institución fundamental en la sociedad a través de sus funciones. Madrid: CEU Ediciones.

Bauman, Z. (2011): Amor líquido. Madrid: Fondo de Cultura Económica.

Catalá Rubio, S. (coord.) (2006): Evolución del Derecho de familia en Occidente. Cuenca: Universidad de Castilla-La Mancha.

Chapman, G. (2011): Los cinco lenguajes del amor. Miami, FL: Editorial Unilit.

Cornides, J. (2010): Natural and Un-Natural Law. Nueva York: International Organizations Law Group.

D’ Agostino, F. (2006): Filosofía de la familia. Pamplona: Rialp.

DAvIS, K. (1985): Contemporary Marriage. Nueva York: Russell Sage Foundation.

Donat, P. (2013): La familia como raíz de la sociedad. Madrid: Biblioteca de Autores Cristianos.

Durant, C. (1996): An Education for a Good Life. Grand Rapids, MI: Acton Institute, p. 7.

Felice, F. (2005): Prospettiva «neocon». Soveria Manelli: Rubbettino, pp. 191-194.

GAMBARA, L. (1906): La familia y su evolución histórica. Barcelona: F. Granada.

Girgis, S., ANDERSON, R.T. y GeORGE, R.P. (2012): What is marriage? Nueva York: Encounter Books.

GonzÁlez Rico, N. (2006): Hablemos de sexo con nuestros hijos. Barcelona: Styria.

GuARDinI, R. (2012): Las etapas de la vida. Madrid: Palabra.

HAYEK, F.A. (2006): Derecho, legislación y libertad. Madrid: Unión Editorial.

- (2008): Los fundamentos de la libertad. Madrid: Unión Editorial.

- (2011): La fatal arrogancia. Madrid: Unión Editorial.

Hildebrand, D. (2009): The Nature of Love. South Bend, IN: St. Augustine's Press.

Huerta de Soto, J. (2000): La Escuela Austriaca. Mercado y creatividad empresarial. Madrid: Editorial Síntesis.

- (2004): «La teoría de la eficiencia dinámica». Procesos de Mercado: Revista Europea de Economía Política, vol. 1, n.o 1, pp. 11-71. 
- (2010): Socialismo, cálculo económico y función empresarial. Madrid: Unión Editorial.

Kuby, G. (2012): Die globale sexuelle Revolution. Kisslegg: Fe-Medienverlags.

LEWIS, C.S.: Los cuatro amores, Rialp, Madrid.

Llano, A. (2010): «Papel del estado y derechos de los padres en la educación sexual y reproductiva». Foro. Revista de Ciencias Jurídicas y Sociales, n. ${ }^{-11-12, ~ p p . ~ 319-351 . ~}$

Lorenzo PinAr, F.J. (ed.) (2009): La familia en la historia. Salamanca: Ediciones Universidad de Salamanca.

Martínez-CAlCERRADA, L. (2005): La homosexualidad y el matrimonio. Madrid: Ediciones Académicas.

Martínez Meseguer, C. (2012): La teoría evolutiva de las instituciones. Madrid: Unión Editorial.

Martínez MuÑoz, J.A. (2009): «Igualdad jurídica y género». Anuario de Derechos Humanos, n. 10, pp. 389-433.

Melina, L., Noriega, J. y Pérez-Soba, J.J. (2007): Caminar a la luz del amor. Madrid: Palabra.

Menger, C. (2012): Principios de economía politica. Madrid: Unión Editorial.

MisEs, L. (2011): La acción humana. Madrid: Unión Editorial.

MolinARI, G. (2006): «Sobre la producción de seguridad». Procesos de Mercado: Revista Europea de Economía Politica, vol. 3, n. ${ }^{-}$ 1, pp. 219-236.

Nogales Lozano, F. (2012): «La educación desde la perspectiva de la Escuela Austriaca de Economía». Procesos de Mercado: Revista Europea de Economía Política, vol. 9, n.o 1, pp. 155-210. Noriega, J. (2007): El destino del eros. Madrid: Palabra.

- (2012): No solo de sexo... Burgos: Monte Carmelo.

NovaK, M. (1996): Business as a Calling. Nueva York: The Free Press. (2013): The Myth of Romantic Love and Other Essays. New Brunswick y Londres: Transaction Publishers.

Roback Morse, J. (2005): Smart sex. San Marcos, CA: Ruth Institute Books.

- (2008): Love E economics. San Marcos, CA: Ruth Institute Books.

- (2009): «The limited-government case for marriage», en Indivisible. Washington, DC: The Heritage Foundation. 
- (2013): The Socialist Attack on the Family. San Marcos, CA: Ruth Institute.

Rodrigo López, M.J., Palacios, J. (coord.) (1998): Familia y desarrollo humano. Madrid: Alianza Editorial.

RojAs, E. (2008): El amor inteligente. Madrid: Temas de Hoy.

Rosmini, A. (1997): Personalismo liberale. Catanzaro: Rubbettino.

SORIANO, D. (2012): «El sistema educativo» en RALLO, J.R.: Un modelo realmente liberal. Madrid: Lid editorial.

Toceueville, A. (2005): La democracia en América. Madrid: Alianza Editorial.

Trillo-FigueroA, J. (2008): Una tentación totalitaria: Educación para la Ciudadanía. Pamplona: Eunsa.

- (2009): La ideología de género. Madrid: Libros Libres.

ViVES, L. (2011): «La familia, minoría creativa», en GrANADOS, L. y De Ribera, I. (eds.): Minorías creativas. Burgos: Monte Carmelo.

West, E.G. (1994): La Educación y el Estado. Madrid: Unión Editorial.

Wojtyla, K. (2008): Amor y responsabilidad. Madrid: Ediciones Palabra.

ZanotTI, G.J. (2011): Antropología filosófica cristiana y economía de mercado. Madrid: Unión Editorial. 